\title{
Duality symmetry and its breakdown in the vicinity of the superconductor-insulator transition
}

\author{
Maoz Ovadia, David Kalok* , Benjamin Sacépé ${ }^{\dagger}$ and Dan Shahar
}

The superconductor-insulator transition (SIT) is an accessible quantum phase transition ${ }^{1,2}$ that is observed in a number of systems and can be driven by various experimental means ${ }^{3-9}$. A central outstanding issue regards the physical nature of the insulating phase terminating superconductivity ${ }^{10}$. Theoretical advances led to the proposition that this insulator is a new state of matter, termed a superinsulator ${ }^{11,12}$, because its properties can be inferred from the superconductor by invoking duality symmetry ${ }^{13}$. Here we report on the observation of duality symmetry near the magnetic-field-driven SIT in amorphous indium oxide. However, we show that the symmetry is broken by the emergence of the strong insulating state at low temperature.

For the magnetic-field-driven SIT ( $B$-SIT) in disordered films, the concept of vortex-charge duality was investigated in ref. 14, using the dirty boson model. In the superconductor, Cooper pairs are condensed into a superfluid at low temperature $(T)$ values leading to a zero resistivity $(\rho)$ state, with vortices as bosonic excitations introducing dissipation and causing finite $\rho$. According to the duality concept, in the insulating state, vortices are condensed in a collective mode with zero conductivity $(\sigma)$, and the Cooper pairs are the bosonic excitation contributing to a finite $\sigma$.

The vortex-charge duality has also been applied to the analysis of Josephson junction arrays ${ }^{15,16}$, which are often used as a model system for the SIT in disordered films ${ }^{11,12,17}$. In these systems the insulating state is commonly ascribed to a Coulomb blockade of superconducting islands. Duality is also applied to many other systems, for example, boson duality in two-dimensional electron gases $^{18}$. Recently, evidence for the vortex-charge duality near a SIT was found in $\mathrm{LaAlO}_{3} / \mathrm{SrTiO}_{3}$ interfaces ${ }^{13}$.

Here, we experimentally investigate the duality symmetry across the $B$-SIT. We apply a duality transformation relating states within the superconductor to states in the $B$-driven insulator. We observe vortex-charge duality symmetry that holds up to one order of magnitude in $B, T$ and $\rho$. The new aspect of this work is that we find systematic deviations from duality symmetry that originate in the $B$-induced magnetoresistance $(\rho(B))$ peak ${ }^{5}$. These deviations are qualitatively different at low and high $T$, as will be shown in detail below.

In Fig. 1 we present $\rho(B)$ isotherms obtained from sample RAM005b, which is superconducting at $B=0$ with $T_{\mathrm{c}}=1.3 \mathrm{~K}$. For all $T$ values where reliable Ohmic data could be collected $\rho$ increases with $B$ until it reaches a $T$-dependent peak at $B_{\text {peak }}$ between 8.5-9.7 T. We restrict ourselves to $T>0.15 \mathrm{~K}$ because in the insulating phase, at $T<0.15 \mathrm{~K}$, severe bi-stability of the electron $T$ develops ${ }^{19}$, resulting in strongly nonlinear $I-V$, that prevented reliable measurements of $\rho$.

The isotherms exhibit a weakly $T$-dependent crossing point close to $h /(2 e)^{2}$, where $h$ is Planck's constant and $2 e$ is the charge

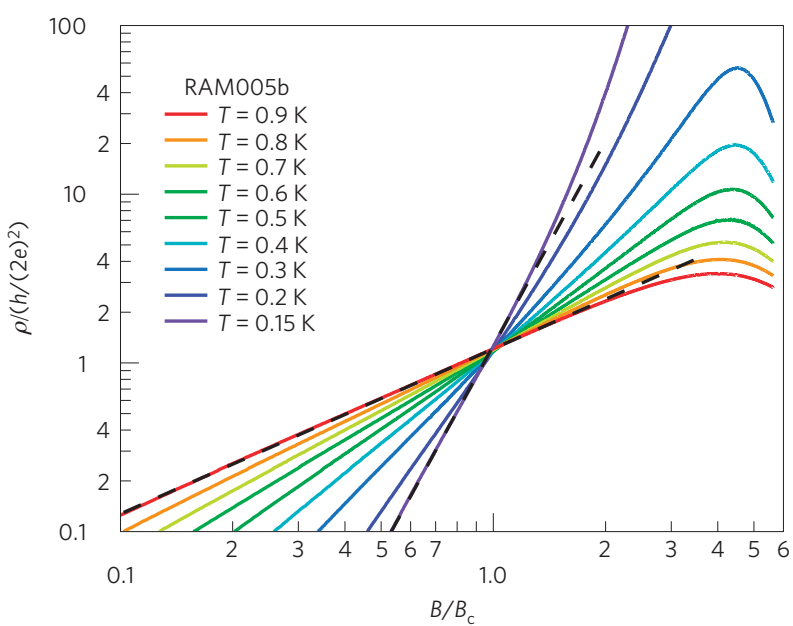

Figure 1 | Magnetic-field-driven SIT. $\rho\left(B / B_{\mathrm{C}}\right)$ isotherms $\left(B_{\mathrm{C}}=2.13 \mathrm{~T}\right)$ of sample RAM005b in $B_{\perp}$ in a log-log plot. The dashed lines along the isotherms $(T=0.9$ and $0.3 \mathrm{~K})$ are guides to the eye following power laws in $B$.

of a Cooper pair, as expected within the bosonic description of the SIT (ref. 14). Therefore, we use the experimental values of $\rho_{\mathrm{c}}=7.8 \pm 0.6 \mathrm{~K} \Omega$ for our analysis of duality. We have chosen a double-logarithmic plot to emphasize the power law that the isotherms follow (dashed lines).

In the vicinity of the $B$-SIT, $\rho$ can be phenomenologically described by a power law:

$$
\rho(B, T)=\rho_{\mathrm{c}}\left(\frac{B}{B_{\mathrm{c}}}\right)^{T_{0} / 2 T}
$$

where $B_{\mathrm{c}}$ is the critical field, separating the superconducting phase and the insulating phase, determined by the crossing point of the $\rho(B)$ isotherms (Fig. 1 ), $\rho_{\mathrm{c}}=\rho\left(B_{\mathrm{c}}\right)$ and $T_{0}$ is a samplespecific parameter ${ }^{20}$. In the superconducting regime, this powerlaw behaviour is consistent with the collective-pinning model of transport in thin films ${ }^{21,22}$ and may indicate the central role played by vortices in our system. The fact that the power law persists above $B_{\mathrm{c}}$ into the insulating state was taken in ref. 20 to suggest that the mechanisms determining transport in the superconductor are also present in the insulating state.

We now show that, under the appropriate selection of parameters, equation (1), and the data in Fig. 1, describe vortexcharge duality-symmetry-relating states in the superconductor to states in the insulator. In a vortex-charge duality transformation one exchanges the roles of vortices and charges or, experimentally, 


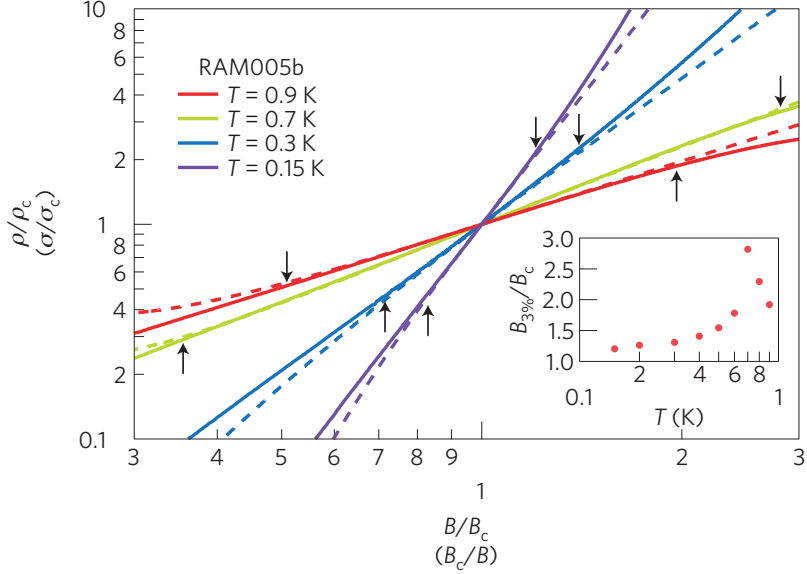

Figure 2 | Duality symmetry. Results of the duality transformation according to equation (2). The solid lines show $\rho / \rho_{\mathrm{c}}$ versus $B / B_{\mathrm{c}}$ isotherms; the dashed lines show the corresponding $\sigma / \sigma_{\mathrm{c}}$ versus $B_{\mathrm{c}} / B$ isotherms. The arrows indicate deviations between $\rho / \rho_{\mathrm{c}}$ and $\sigma / \sigma_{\mathrm{c}}$ of $3 \%$. Inset: $B / B_{\mathrm{c}}$ values of the determined $3 \%$ deviations versus $T$.

currents $(I)$ and voltages $(V)$. Considering the practical definitions of $\sigma=I / V$ and $\rho=V / I$, this is tantamount to $\sigma \leftrightarrow \rho$. To guarantee self-duality at the transition point ${ }^{14}$, we theoretically require that $\rho_{\mathrm{c}}=h /(2 e)^{2}$. We therefore adhere to expressing $\rho$ in units of $\rho_{\mathrm{c}}$.

To relate the two phases we need to map $\rho$ in the superconductor $\left(B_{\mathrm{sc}}<B_{\mathrm{c}}\right)$, onto $\sigma$ in the insulator $\left(B_{\text {ins }}>B_{\mathrm{c}}\right)$. The requirement for vortex-charge duality symmetry is, therefore,

$$
\rho\left(B_{\mathrm{sc}}\right)=\sigma\left(B_{\text {ins }}\right)
$$

for the appropriate choice of $B_{\mathrm{sc}}$ and $B_{\text {ins }}$

To find the set of $B$ values $\left(B_{\mathrm{sc}}, B_{\text {ins }}\right)$ where these dual states exist we recall that the power law of equation (1) extends into the insulator and therefore

$$
\begin{gathered}
\rho=\left(B_{\mathrm{sc}} / B_{\mathrm{c}}\right)^{T_{0} / 2 T} \\
\sigma=1 / \rho=\left(B_{\mathrm{c}} / B_{\text {ins }}\right)^{T_{0} / 2 T}
\end{gathered}
$$

leading to the relation

$$
\frac{B_{\mathrm{sc}}}{B_{\mathrm{c}}}=\frac{B_{\mathrm{c}}}{B_{\text {ins }}}
$$

To test for duality symmetry we re-plot, in Fig. 2, several of the isotherms from Fig. 1 along with the corresponding dual curves transformed as dictated by equations (2) and (3). The set of $\rho$ curves is plotted against $B / B_{\mathrm{c}}$ and the calculated $\sigma$ curves are plotted using the inverse axis, $B_{\mathrm{c}} / B$.

As seen by the overlap of each pair of curves, duality symmetry holds. Its regime of applicability, indicated by arrows on the curves where deviations exceed $3 \%$, extends to $B / B_{\mathrm{c}}=0.3-3, T=0.3-0.9 \mathrm{~K}$ and $\rho / \rho_{\mathrm{c}}=0.1-10$. Further away from $B_{\mathrm{c}}$ deviations from duality symmetry become visible. We shall discuss these deviations below.

The physics that links the pairs of $B$ values where $\rho$ and $\sigma$ obey duality symmetry was discussed in ref. 14. In a type-II superconductor, the vortex density is given by $n_{v}=B / \phi_{0}$, with $\phi_{0}=h / 2 e$ being the superconducting flux quantum. Above a certain critical $n_{v}, n_{v}^{c}=B_{c} / \phi_{0}$, the vortices form a condensate, which renders the electronic system insulating. At finite $T$ values, thermally excited Cooper pairs lead to a finite $\sigma$, in a dual way to the dissipation caused by thermally excited vortices in the superconductor ${ }^{22}$. We note that, theoretically, vortex-charge

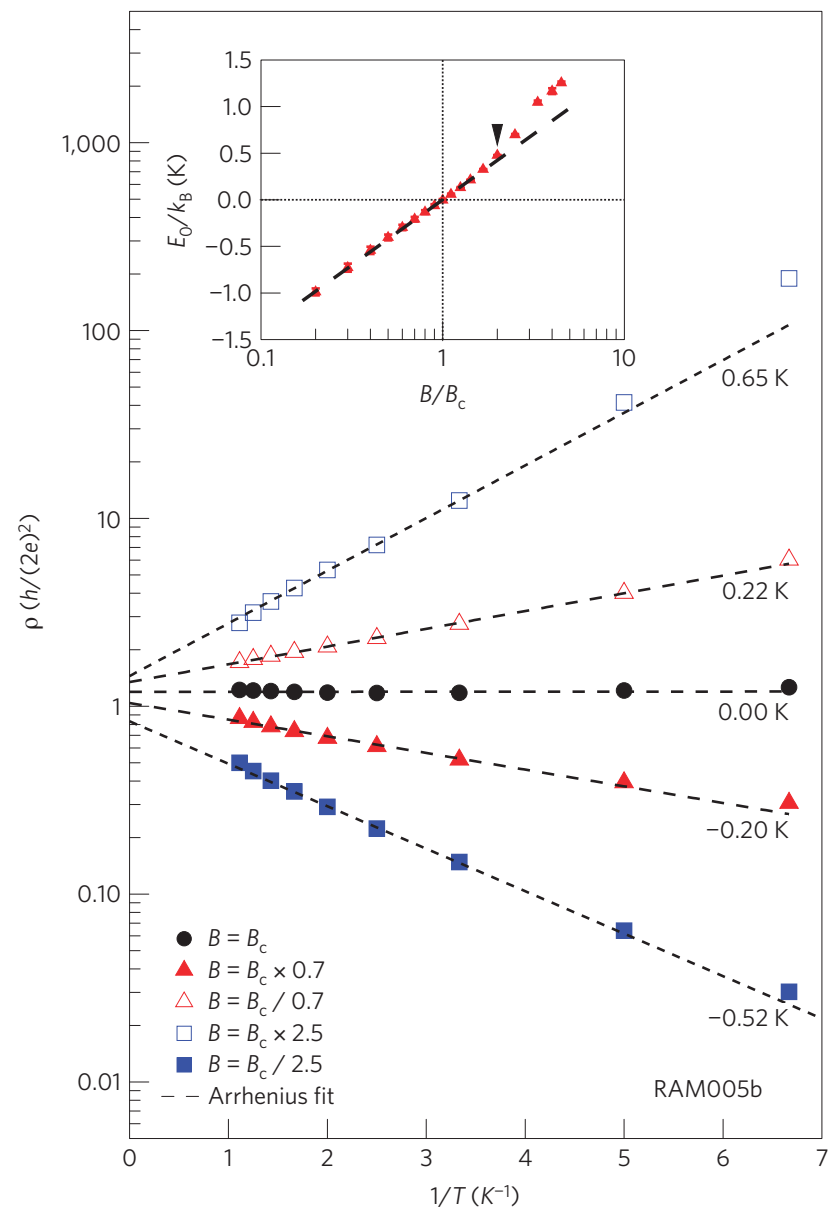

Figure 3 | Magnetic-field-dependent activated transport. $\rho$ versus $1 / T$ at selected dual $B$ values determined by equation (3). Dashed lines indicate Arrhenius fits, $\rho(T, B)=\rho_{\mathrm{c}} \exp \left(E_{0}(B) / T\right)$. The corresponding $E_{0}$ is shown adjacent to each line. Inset: extracted $E_{0}$ versus $B / B_{c}$. The dashed line indicates $E_{0} \propto \ln \left(B / B_{c}\right)$. The arrow indicates $B / B_{c}=2$, where $E_{0}$ exceeds its expected dual value.

duality symmetry is expected to hold only close to the self-dual point at $B_{\mathrm{c}}$ (refs 14,15) and deviations should occur when moving away from $B_{\mathrm{c}}$.

In addition, duality symmetry is expected to hold only if the Coulomb repulsion between the Cooper pairs in the insulator will be similar to the vortices interaction in the superconducting phase, which is logarithmic. This is feasible if the Cooper pairs reside in a medium that will confine their electric field lines to two dimensions. It has been suggested that, near a conductivity transition, the expected divergence of the dielectric constant may provide such conditions. This possible divergence is amenable to experimental tests. The thickness of our samples is of the order of the estimated superconducting coherence length rendering them borderline two-dimensional.

We now turn to the analysis of the breakdown of duality symmetry. In our phenomenological interpretation, duality symmetry stems from the power-law $\rho(B)$ dependence, and the breakdown of duality symmetry is associated with deviations from this power law. Inspecting Fig. 1 it is obvious that the data systematically deviate from the power law (dashed lines) only in the insulating regime. At high $T(>0.7 \mathrm{~K})$ in the insulating regime the power law overestimates the measured $\rho$ and at lower $T$ it underestimates it. Below $0.7 \mathrm{~K}$ the deviations get larger and move to lower $B$ as $T$ is lowered. Whereas the high- $T$ deviations can be qualitatively accounted for by additional thermal, for example, 


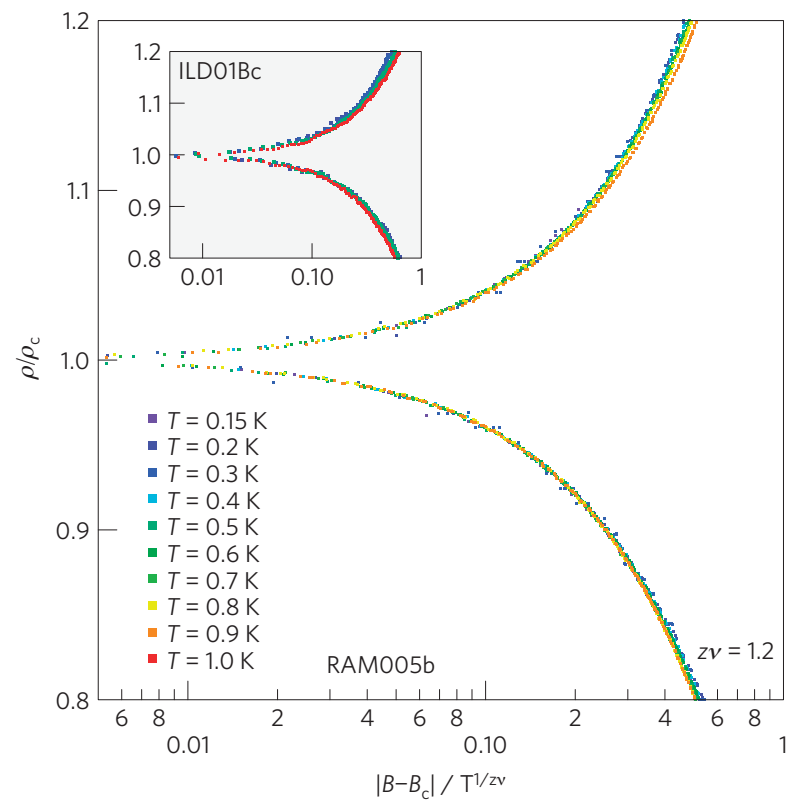

Figure 4 | Scaling. Temperature scaling for samples RAM005b and ILD01BC (inset). The shown exponent $z v=1.2$ is determined by the best data collapse.

single-electron, excitations increasing $\sigma$, the low- $T$ deviations cannot be similarly explained.

The central new aspect of this study is that these low- $T$ deviations from duality symmetry herald the arrival of the insulating state. This is in contrast to the theoretical framework of refs $11,12,17$ in which the high- $B$ insulating state, termed a superinsulator ${ }^{11,12}$, is taken to be a dual state of the superconducting phase. We note that in these models, and in Josephson junction arrays (ref. 16), duality is related to the ratio $E_{\mathrm{J}} / E_{\mathrm{C}}$, where $E_{\mathrm{J}}$ is the Josephson coupling between superconducting islands and $E_{\mathrm{C}}$ is the charging energy, and not directly to $B$. However, from $T=0.9$ to $0.7 \mathrm{~K}$ duality symmetry seems to become better with lower $T$, consistent with vortex-charge duality. In contrast, the insulating state below $T=0.7 \mathrm{~K}$ breaks duality symmetry systematically more strongly with lower $T$, and is no longer related to the $T$ and $B$ evolution of the superconductor. We can separate these two regimes phenomenologically by the value of the $\rho_{\text {peak }}$ : it is below $h / e^{2}$ at high $T$ and above $h / e^{2}$ at low $T$. This behaviour cannot be explained by present theoretical models.

More light can be shed on the source of the deviations from duality by inspecting the $T$ dependence of $\rho(B)$. When the power law of equation (1) is written in an exponential form

$$
\rho(T, B)=\rho_{\mathrm{c}} \exp \left(\frac{E_{0}(B)}{k_{\mathrm{B}} T}\right)
$$

it reveals an Arrhenius behaviour with activation energy $E_{0}=\left(k_{B} T_{0} / 2\right) \ln \left(B / B_{c}\right)$. Theoretically this form stems from the collective-pinning model for vortex transport although, obviously, it is applicable only for $B<B_{c}$ (ref. 5). Viewed this way, duality symmetry translates into a symmetry in the activation energy:

$$
E_{0}\left(B_{\mathrm{sc}}\right)=-E_{0}\left(B_{\text {ins }}\right)
$$

Examining Fig. 3 , where we plot $E_{0}$ versus $B / B_{\mathrm{c}}$ (inset), we observe indeed the symmetry of equation (4) (dashed line) in the extracted $E_{0}(B)$ for $B / B_{\mathrm{c}} \leq 2$ supporting vortex-charge duality near $B_{\mathrm{c}}$. The main observation can be seen in Fig. 3 for $B / B_{\mathrm{c}}>2$, $E_{0}$ exceeds the extrapolation of $\ln \left(B / B_{\mathrm{c}}\right)$, indicated by the arrow,

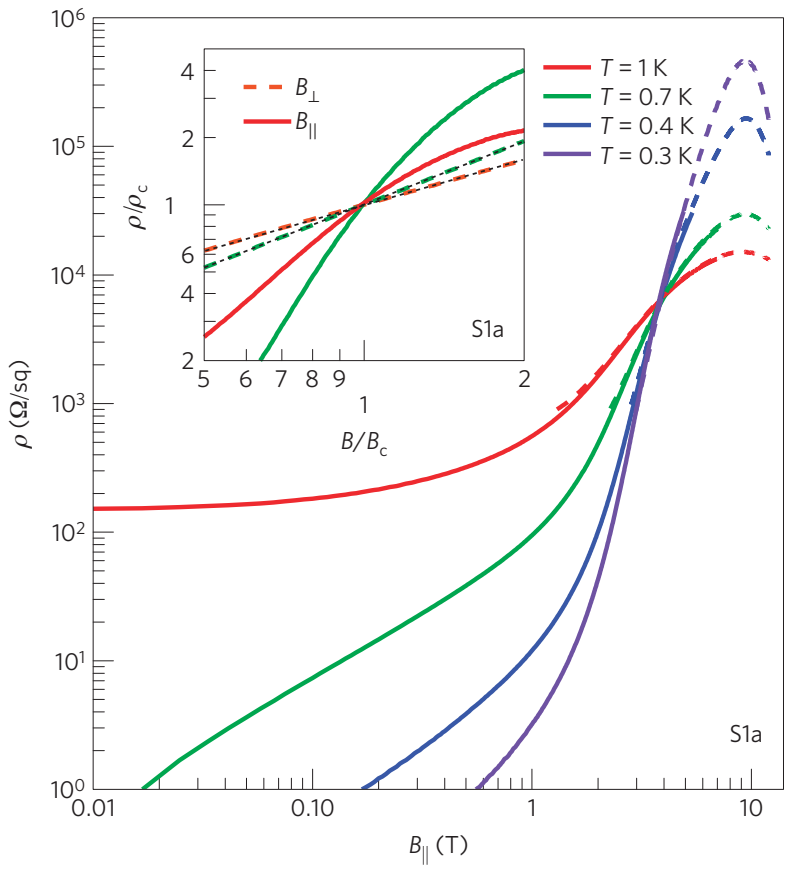

Figure $\mathbf{5}$ | SIT in a parallel magnetic field. $\rho(B)$ isotherms in $B_{\|}$of sample S1a. Solid lines were measured in a four-point current bias set-up, and the dashed lines in a two-point voltage bias set-up. Inset: comparison of $\rho / \rho_{\mathrm{C}}$ versus $B / B_{\mathrm{c}}$ in $B_{\perp}$ (dashed) and $B_{\|}$(solid). The power law across $B_{\mathrm{c}}$ is indicated by dotted lines along the curves.

breaking duality. This stronger insulating behaviour for $B / B_{c}>2$ violates duality symmetry already in the activated transport regime (compare $R$ versus $T$ in Fig. 3 ) and therefore cannot be explained by a low- $T$ transition to a superinsulating state ${ }^{11,12}$.

Near a quantum phase transition, criticality results in scaling laws $^{1,4}$. For the SIT, this was demonstrated for many systems although the critical exponent, $z v$, describing the transition lacks the expected universal value. In Fig. 4 we show the result of a scaling analysis we performed on the $\rho$ data from samples RAM005b and ILD01Bc. We obtained a good collapse of the data with the resulting $z v=1.2 \pm 0.1$, consistent with previous findings ${ }^{4}$.

One of the obvious strengths of the scaling approach is that important information about the physics of the system can be gathered from measurements even without a complete microscopic theory. By conducting scaling analysis near the critical point, the experimentalist can obtain profound symmetry classification of the system without full knowledge of the functional form the data follow. In our system, however, the power law depicted in Fig. 1 can be regarded as a measurement of the entire scaling function. This awaits further tests in other systems as well as better theoretical understanding.

As a further test we measure $\rho(B)$ in a parallel $B$-orientation $\left(B_{\|}\right)$. If vortices are behind the power law in $B$, it will be absent in $B_{\|}$ because vortices are not expected in $B_{\|}$in our thin films. In Fig. 5 we show $\rho\left(B_{\|}\right)$of sample S1a, exhibiting a SIT similar to RAM005 in $T, B$ and $\rho$ range. No power law is visible across the SIT in $B_{\|}$. We note that in the superconductor a power law is visible $\left(B_{\|}<1 \mathrm{~T}\right.$ and $T \leq 0.7 \mathrm{~K}$ ) but this can be explained by an angular misalignment of $0.6^{\circ}$ in $B$-orientation, that is, within the experimental accuracy.

To show that the power law in $B_{\perp}$ is real and not an artefact of the log-log plot, we re-plot in the inset of Fig. $5 \rho / \rho_{\mathrm{c}}$ versus $B / B_{\mathrm{c}}$ of selected $B_{\|}$isotherms (solid lines) with the corresponding $B_{\perp}$ isotherms (dashed lines). Whereas the $B_{\perp}$ data follows the power law across $B_{\mathrm{c}}$ (dotted lines along the curves), the $B_{\|}$data show no power law. 
Our observation of duality symmetry suggests a comparison with the charge-flux duality observed near the quantum-Hall liquid to insulator transition ${ }^{18}$. The duality in the quantum-Hall regime is associated with the Landau-level filling factor, determined by the quantization of the Hall conductivity, whereas near the SIT the relevant parameter is the fraction $n_{v} / n_{v}^{c}$, determined by the penetration of one vortex per $\phi_{0}$ of flux.

Finally, an analogy between the SIT driven by disorder and $B$ can be made ${ }^{23}$. Increasing the number of Cooper pairs by decreasing the disorder leads to the formation of the Cooper-pair condensate, the superconductor. Increasing the vortex density by increasing $B$ leads to a vortex condensate, the insulating state. Following this relationship, the experiments on the $B$-SIT and the experiments tuning the carrier density form a dual set of experiments. Interestingly, this analogy implies that the disorder-driven SIT at $B=0$ must have a different mechanism for the insulating state; a distinction that cannot be made from experiments: Transport and scanning tunnelling experiments ${ }^{24-26}$ as well as microscopic model calculations ${ }^{27,28}$ suggest that Cooper pairs persist also in the disorder-driven insulating state.

\section{Methods}

Samples. The samples RAM005b, ILD01Bc and S1a were grown by electron-gun evaporation of $99.99 \%$-purity sintered $\mathrm{In}_{2} \mathrm{O}_{3}$ targets onto a silicon/silicon dioxide substrate, resulting in 30-nm-thick amorphous indium oxide films. The geometry was defined by a shadow mask and electric contacts were made using pressed indium and gold wires.

Measurements. Four-probe resistance measurements (2.5 squares in series) were performed using standard lock-in techniques with excitation currents of 1-10 nA and, for sample S1a, also in a two-probe voltage bias set-up $(V=50 \mu \mathrm{V} ; 5$ squares in series). The samples were cooled in a dilution refrigerator with a base temperature of $0.01 \mathrm{~K}$. Magnetic fields were applied perpendicular to the sample surface. S1a was also investigated in parallel magnetic fields.

Received 19 December 2012; accepted 19 April 2013; published online 2 June 2013

\section{References}

1. Sondhi, S. L., Girvin, S. M., Carini, J. P. \& Shahar, D. Continuous quantum phase transitions. Rev. Mod. Phys. 69, 315-333 (1997).

2. Shangina, E. L. \& Dolgopolov, V. T. Quantum phase transitions in two-dimensional systems. Phys. Usp. 46, 777-787 (2003).

3. Haviland, D. B., Liu, Y. \& Goldman, A. M. Onset of superconductivity in the two-dimensional limit. Phys. Rev. Lett. 62, 2180-2183 (1989).

4. Hebard, A. F. \& Paalanen, M. A. Magnetic-field-tuned superconductorinsulator transition in two-dimensional films. Phys. Rev. Lett. 65, 927-930 (1990).

5. Sambandamurthy, G., Engel, L. W., Johansson, A. \& Shahar, D. Superconductivity-related insulating behavior. Phys. Rev. Lett. 92, 107005 (2004).

6. Baturina, T. I. et al. Superconductivity on the localization threshold and magnetic-field-tuned superconductor-insulator transition in TiN films. JETP Lett. 79, 337-341 (2004).

7. Parendo, K. A. et al. Electrostatic tuning of the superconductor-insulator transition in two dimensions. Phys. Rev. Lett. 94, 197004 (2005).

8. Bollinger, A. T. et al. Superconductor-insulator transition in $\mathrm{La}_{2-x} \mathrm{Sr}_{x} \mathrm{CuO}_{4}$ at the pair quantum resistance. Nature 472, 458-460 (2011).

9. Allain, A., Han, Z. \& Bouchiat, V. Electrical control of the superconducting-to-insulating transition in graphene-metal hybrids. Nature Mater. 11, 590-594 (2012).
10. Gantmakher, V. F. \& Dolgopolov, V. T. Superconductor-insulator quantum phase transition. Phys. Usp. 53, 1-49 (2010).

11. Vinokur, V. M. et al. Superinsulator and quantum synchronization. Nature 452, 613-615 (2008).

12. Baturina, T. I. \& Vinokur, V. M. Superinsulator-superconductor duality in two dimensions. Ann. Phys. 331, 236-257 (2013).

13. Mehta, M. M. et al. Evidence for charge-vortex duality at the $\mathrm{LaAlO}_{3} / \mathrm{SrTiO}_{3}$ interface. Nature Commun. 3, 955-962 (2012).

14. Fisher, M. P. A. Quantum phase transitions in disordered two-dimensional superconductors. Phys. Rev. Lett. 65, 923-926 (1990).

15. Fazio, R. \& Schön, G. Charge and vortex dynamics in arrays of tunnel junctions. Phys. Rev. B 43, 5307-5320 (1991).

16. Fazio, R. \& van der Zant, H. Quantum phase transitions and vortex dynamics in superconducting networks. Phys. Rep. 355, 235-334 (2001).

17. Fistul, M. V., Vinokur, V. M. \& Baturina, T. I. Collective Cooper-pair transport in the insulating state of Josephson-junction arrays. Phys. Rev. Lett. 100, 086805 (2008).

18. Shahar, D., Tsui, D. C., Shayegan, M., Shimshoni, E. \& Sondhi, S. L. Evidence for charge-flux duality near the quantum Hall liquid-to-insulator transition. Science 274, 589-592 (1996).

19. Ovadia, M., Sacépé, B. \& Shahar, D. Electron-phonon decoupling in disordered insulators. Phys. Rev. Lett. 102, 176802 (2009).

20. Sambandamurthy, G. et al. Power law resistivity behavior in 2D superconductors across the magnetic field-tuned superconductor-insulator transition. Europhys. Lett. 75, 611-617 (2006).

21. Ephron, D., Yazdani, A., Kapitulnik, A. \& Beasley, M. R. Observation of quantum dissipation in the vortex state of a highly disordered superconducting thin film. Phys. Rev. Lett. 76, 1529-1532 (1996).

22. Blatter, G., Feigel'man, M. V., Geshkenbein, V. B., Larkin, A. I. \& Vinokur, V. M. Vortices in high-temperature superconductors. Rev. Mod. Phys. 66, 1125-1388 (1994)

23. Goldman, A. M. \& Markovic, N. Transitions in the two-dimensional limit. Phys. Today 51, 39 (November, 1998).

24. Baturina, T. I., Mironov, A. Y., Vinokur, V. M., Baklanov, M. R. \& Strunk, C. Localized superconductivity in the quantum-critical region of the disorder-driven superconductor-insulator transition in TiN thin films. Phys. Rev. Lett. 99, 257003 (2007).

25. Sacépé, B. et al. Disorder-induced inhomogeneities of the superconducting state close to the superconductor-insulator transition. Phys. Rev. Lett. 101, 157006 (2008).

26. Sacépé, B. et al. Localization of preformed Cooper pairs in disordered superconductors. Nature Phys. 7, 239-244 (2011).

27. Feigel'man, M. V., Ioffe, L. B., Kravtsov, V. E. \& Cuevas, E. Fractal superconductivity near localization threshold. Ann. Phys. 325, 1390-1478 (2010).

28. Bouadim, K., Loh, Y. L., Randeria, M. \& Trivedi, N. Single- and two-particle energy gaps across the disorder-driven superconductor-insulator transition. Nature Phys. 7, 884-889 (2011).

\section{Acknowledgements}

We thank M. Feigel'man, B. Altshuler, S. Mirlin, I. Aleiner, Y. Oreg and Z. Ovadyahu for discussions. This work was supported by the Minerva foundation with funding from the Federal German Ministry for Education and Research.

\section{Author contributions}

All authors contributed equal parts to the paper. D.S. initiated this work.

\section{Additional information}

Reprints and permissions information is available online at www.nature.com/reprints. Correspondence and requests for materials should be addressed to D.K.

\section{Competing financial interests}

The authors declare no competing financial interests. 